\title{
A bulbar artery pseudoaneurysm following traumatic urethral catheterization
}

\author{
Mathieu Bettez, MD; ${ }^{1}$ Melanie Aubé, MD; ${ }^{2}$ Mohamed El Sherbiny, MD; Tatiana Cabrera, MD; ${ }^{2}$ \\ Roman Jednak, $M D^{\prime}$
}

'Division of Pediatric Urology, Montreal Children's Hospital, McGill University Health Centre, Montreal, QC, Canada; ${ }^{2}$ Department of Radiology, Royal Victoria Hospital, McGill University Health Centre, Montreal, QC, Canada

Cite as: Can Urol Assoc J 2017;11(1-2):E47-9. http://dx.doi.org/10.5489/cuaj.4050 Published online January 12, 2017

\section{Abstract}

Traumatic urethral catheterization may result in a number of serious complications. A rare occurrence is the development of a urethral pseudoaneurysm. We report the case of a 13-year-old male who required placement of a Foley catheter for an orthopedic surgical procedure. The Foley was misplaced in the bulbourethra, resulting in the development of a bulbar artery pseudoaneurysm. Profuse bleeding via the urethra was noted after removal of the catheter, and the patient experienced severe intermittent hematuria during the postoperative period. Cystoscopy revealed a pulsatile mass within the bulbourethra. Angiography confirmed a bulbar artery pseudoaneurysm, which was successfully embolized with resolution of bleeding.

\section{Introduction}

The insertion of a urethral catheter is a frequently performed procedure that is not without risk. The improper insertion of a catheter can result in false passage, urethral stricture, fistula, and pseudoaneurysm. The latter, although a rare complication, can be devastating.

In urology, pseudoaneurysms may occur more commonly in the kidney and the pelvic vessels, either post-traumatically or iatrogenically following surgery. We report the case of a pseudoaneurysm of the bulbar artery after traumatic insertion of a urethral catheter.

\section{Case report}

A 13-year-old male was admitted for elective orthopaedic surgery. Although he had undergone numerous prior surgical procedures for arthrogryposis multiplex congenita (AMC), his only prior urological event was a urinary tract infection at the age of seven. There was no personal or family history of a bleeding disorder. A 14-French (Fr) Foley catheter with a 10 cc balloon was placed, following induction of general anaesthesia, to monitor urine output during the case. Intraoperatively, no urine drained via the catheter throughout the entire four-hour case. Following the procedure, the catheter was removed, as it was assumed to be misplaced. Upon its removal, there was significant bleeding per urethra with subsequent clot retention. A 14-Fr silicone catheter was easily reinserted and the patient was manually declotted. Despite declotting, hematuria persisted and additional declotting was periodically required over the next 24 hours. The immediate postoperative hemoglobin was $110 \mathrm{~g} / \mathrm{L}$. Hematuria resolved by the following day. Repeat hemoglobin on postoperative Day 1 was at $82 \mathrm{~g} / \mathrm{L}$. Significant urethral bleeding recurred 48 hours later, which resolved following a catheter change (upscaled to 18-Fr) and manual declotting.

Ten days following the initial event, the catheter was removed with the clinical hypothesis that a false passage was the likely cause of bleeding. The following day the patient once again bled significantly with an associated drop in the hemoglobin to $62 \mathrm{~g} / \mathrm{L}$. Transfusion was refused for religious reasons. An urgent cystoscopic examination was performed given the severity and atypical pattern of bleeding.

Cystoscopy revealed clot in the left wall of the bulbar urethra within a mucosal defect. A urethral catheter was left in place and removed four days later. Urethral bleeding recurred, but resolved within 30 minutes in response to perineal compression. The hemoglobin dropped to 48 $\mathrm{g} / \mathrm{L}$ and the patient developed tachycardia. The patient was transfused, after informed consent, and cystoscopy was repeated. A pulsatile mass was identified in the left lateral wall of the bulbar urethra, which at the time was not actively bleeding (Fig. 1).

A pseudoaneurysm was suspected and urgent angiography of the left internal iliac artery was performed through a right common femoral approach. This confirmed the diagnosis of a pseudoaneurysm of the left bulbar artery (Fig. 2A). Superselective embolization of the feeding vessel was 


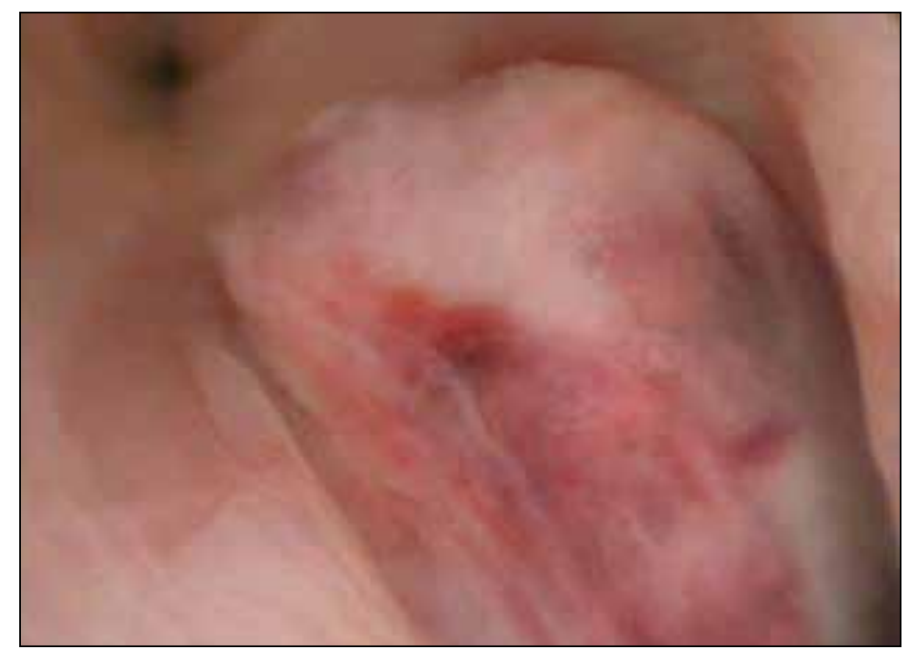

Fig. 1. Pulsatile mass within the bulbar urethra, as seen during cystoscopy.

performed using gelfoam and a $2 \mathrm{~mm}$ Vort $\mathrm{X}^{\circledR}$ metallic coil (Boston Scientific, Natick, MA, U.S.) resulting in immediate interruption of blood flow to the pseudoaneurysm (Fig. 2B). The procedure was completed without any complication. The hematuria resolved immediately, and the hemoglobin improved. The patient was discharged 24 hours later. There have been no episodes of bleeding within a two-month followup period since embolization.

\section{Discussion}

A pseudoaneurysm is defined as a "disruption of an artery with patent flow in a defined space beyond the confines of the vessel." ${ }^{1}$ It is the result of a subtotal injury to the adventitia of the vessel, with protrusion of the intima and the media that creates a pressurized sac causing a pulsatile hematoma. This can be caused by a trauma, inflammation or, more commonly, iatrogenically. ${ }^{2}$ A pseudoaneurysm can

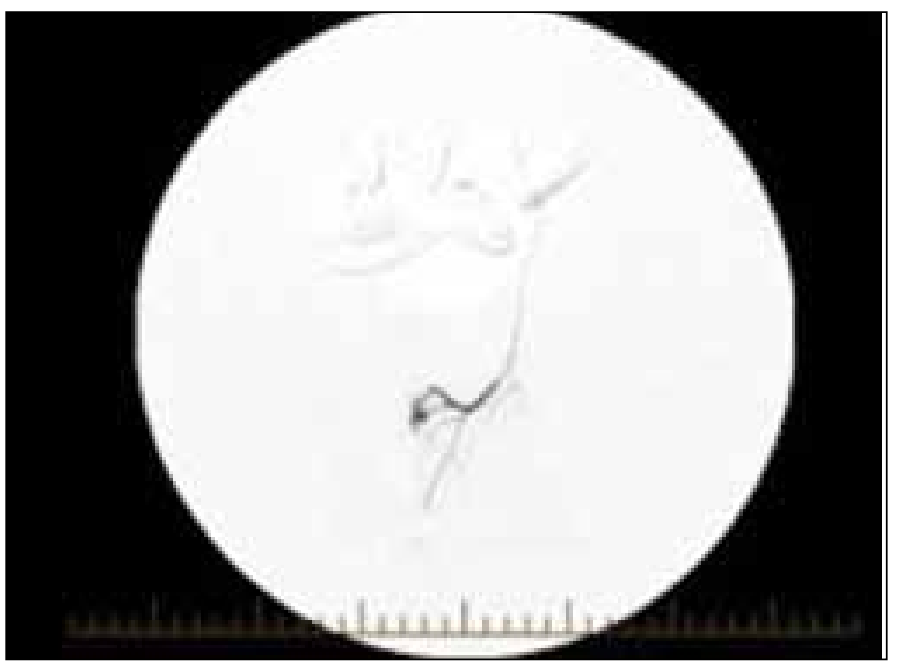

Fig. 2A. Subtraction angiography images of bulbar artery pseudoaneurysm. form in any blood vessel and the presentation will depend on the location and size of the lesion. Pseudoaneurysms following percutaneous nephrolithotomy, partial nephrectomy, radical nephrectomy, radical prostatectomy, radical cystectomy, and abdominal trauma have all been reported in the urological literature. ${ }^{2}$ A single case of a bulbar pseudoaneurysm has been reported in an adult. ${ }^{3}$

The symptomatology of pseudoaneurysms is variable. The natural history is thrombosis, progression or rupture. ${ }^{2}$ When the bulbar artery is affected, the patient can present with intermittent hematuria or urethrorrhagia. Initially, it can be mistaken for bleeding related to a false passage. When bleeding persists despite adequate conservative management, this should raise suspicion of a urethral pseudoaneurysm. Bleeding with this pathology can often be temporarily controlled by perineal compression. It has been hypothesized that the traumatic injury to the arterial wall is actually pressure necrosis produced by catheter balloon inflation within the urethra. ${ }^{3}$ The etiology is plausible, but speculative and the exact cause for pseudoaneurysm formation remains unknown. Nevertheless, inflation of a urethral catheter balloon in the bulbar urethra for a prolonged period of time appears to be a risk factor for the development of a bulbar artery pseudoaneurysm.

The diagnosis of a pseudoaneurysm is typically made by radiological imaging in the form of a Doppler ultrasound, an angio-computed tomography (CT) scan, or by angiography, which remains the gold standard. We report the first case of a bulbar artery pseudoaneurysm in a child diagnosed by cystoscopy. Angiography remains the primary diagnostic and a therapeutic procedure for pseudoaneurysms. It is the most sensitive procedure and can determine the presence of collateral vascularisation, as well as evaluate the diameter of the neck of the pseudoaneurysm. The disadvantages of angiography are the potential for contrast-induced nephrotoxicity

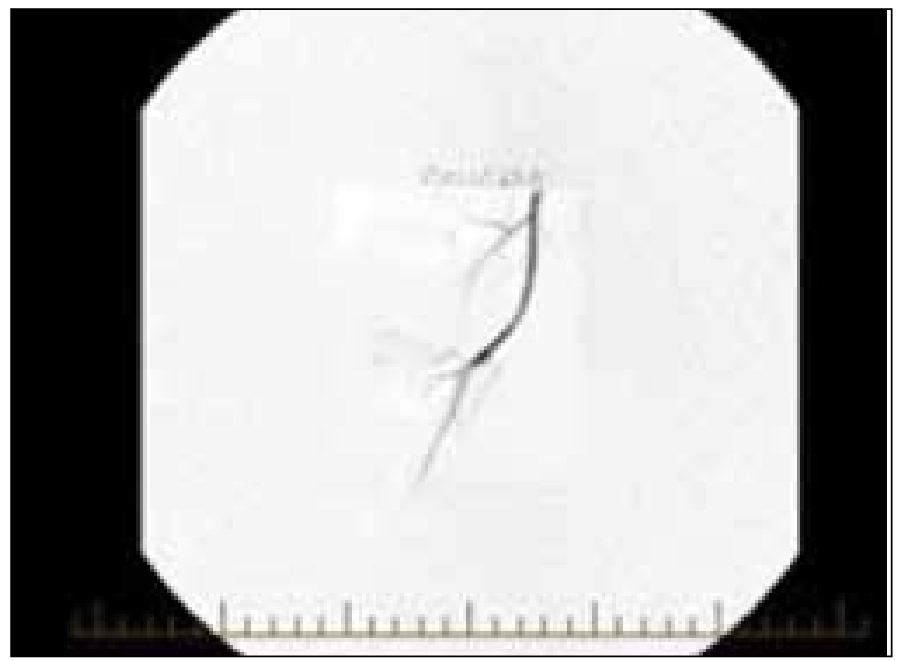

Fig. 2B. Interruption of flow to the pseudoaneurysm following selective occlusion of the afferent vessel. 
and its invasive nature. ${ }^{2}$ The complications are dependent on operator experience, vascular territory affected, the specific lesion addressed, and the clinical condition of the patient. ${ }^{4}$ The procedure has been associated with sepsis, abscess formation, non-target embolization, haemorrhage and, even, mortality. ${ }^{4} \mathrm{~A}$ pseudoneurysm needs to be treated when it is symptomatic, not resolving with conservative management or enlarging. ${ }^{2}$ We speculate that possible risks of urethral pseudoaneurysm embolization are erectile dysfunction and urethral stricture due to necrosis. The limited number of cases reported to date makes this impossible to determine.

\section{Conclusion}

Prolonged inflation of a urethral catheter in the bulbar urethra can result in a urethral arterial pseudoaneurysm. The presenting symptom of this condition is intermittent urethrorragia. Bleeding may be significant. In a clinically stable patient with an unclear diagnosis, cystoscopy may adequately diagnose this condition. Profuse, uncontrolled bleeding should prompt rapid angiography to confirm the diagnosis and treat the condition by embolization.
Competing interests: The authors report no competing personal or financial interests.

This paper has been peer-reviewed.

\section{References}

1. Kapoor BS, Haddad HL, Saddekni S, et al. Diagnosis and management of pseudoaneurysms: An update. Curr Probl Diagn Radiol 2009;38:170-88. https://doi.org/10.1067/i.cpradiol.2008.11.001

2. Sutherland DE, Williams SB, Rice D, et al. Vascular pseudoaneurysms in urology: Clinical characteristics and management. J Endourol 2010;24:915-21. https://doi.org/10.1089/end.2009.0662

3. Radhakrishnan S, Marsh R, Sheikh N, et al. Urethral catheter induced pseudoaneurysm of the bulbar artery. Int J Urol 2005;12:922-4. https://doi.org/10.1111/i.1442-2042.2005.01176.x

4. Angle JF, Siddiqi NH, Wallace MJ, et al. Quality improvement guidelines for percutaneous transcatheter embolization: Society of interventional radiology standards of practice committee. J Vasc Interv Radiol 2010;21:1479-86. https://doi.org/10.1016/i.jvir.2010.06.014

Correspondence: Dr. Melanie Aubé, Montreal Children's Hospital, Montreal, QC, Canada; Melanie.aube-peterkin@mail.mcgill.ca 